\title{
Quad Difference Labeling for Mechanical Process Operating at Repetitive Cycles
}

\author{
G. Subashini
}

\begin{abstract}
In this present work, we discuss the concept of Quad difference labeling $(Q D L)$ behavior of path, cycle and wheel related graphs like ladder, triangular ladder, diagonal ladder, fan, friendship, gear, helm, wheel and $C_{-} n \boldsymbol{O K}$ lgraphs.
\end{abstract}

Keywords: QDL, Quad difference graph $(Q D G), L \_n, \llbracket T L 】$

$\_n, \llbracket D L 】 \_n, F \_n, T \_n, G \_n, H_{-} n, W_{-} n a n d C_{-} n \odot K_{-} 1$.

\section{INTRODUCTION}

As a standard notation, assume that $\mathrm{G}=(\mathrm{V}, \mathrm{E})$ is a finite, simple and undirected graph with $\mathrm{p}$ vertices and $\mathrm{q}$ edges. Terms and terminology as in [4]. A dynamic survey on graph labeling is regularly updated in [2]. [1] proved On square sum graphs. V. Govindan, S. Dhivya proved that Difference labeling of Jewel graph is square difference, cube difference and quad difference labeling[3].The concept of cube difference and square difference labeling was introduced in $[4,5]$. J.Shiama proved that the following graphs paths, cycle, stars and trees admits cube difference labeling.

\section{Definition 2.1}

Let $\mathrm{G}$ be a graph and is said to be QDL if there exist a one to one and onto function from vertices to $\{0,1, \ldots, \mathrm{p}-1\}$ such that $f$ induces the mapping $f^{*}: E(G) \rightarrow N$ is given by $f^{*}(u v)=\left|[f(u)]^{4}-[f(v)]^{4}\right|$ is injective.

\section{Definition 2.2}

Ladder graph: $L_{n}$ is a planar graph with $2 \mathrm{n}$ vertices and $3 \mathrm{n}-2$ edges. Ladder graph is obtained as the Cartesian product of two paths one of which has only one edge which is denoted by $L_{n}=P_{n} \mathrm{x} P_{2}$.

\section{Definition 2.3}

Triangular ladder: $T L_{n}, n \geq 2$ is a graph obtained from ladder by adding the edge $u_{i} v_{i+1}, 1 \leq i \leq n-1$. The vertices of $\mathrm{L}_{\mathrm{n}}$ are $u_{i}$ and $v_{i}$ and are two paths in the graph $\mathrm{L}_{\mathrm{n}}$ where $i=1$ ton.

\section{Definition 2.3}

Diagonal ladder: $D L_{n}, n \geq 2$ is a ladder graph with $2 n$ vertices and is got from a ladder graph with the additional edges $u_{i} v_{i+1}, u_{i+1} v_{i}, 1 \leq i \leq n-1$.

\section{Definition 2.4}

Revised Manuscript Received on December 16, 2019

G. Subashini, Assistant Professor, Department of Science and Humanities, Bharath Institute of Higher Education and Research, India
Fan graph: $F_{n}, n \geq 2$ accomplished by joining all vertices of a path $P_{n}$ to a further vertex called centre and is denoted by $F_{n}=K_{l}+P_{n}$.

Definition 2.5

Friendship graph: a graph which consists of $n$ triangles with common vertex called center.

\section{Definition 2.6}

Wheel graph: $W_{n}$ is join of $C_{n}$ and $K_{l}$ i.e $W_{n}=C_{n}+K_{l}$ here the edges of $C_{n}$ are the rim edges of $W_{n}$.

Definition 2.7

Gear graph: $G_{n}$ is attained from the wheel $W_{n}$ by subdividing each of its rim edges.

\section{Definition 2.8}

Helm graph: $H_{n}$ is a graph acquired from the $W_{n}$ by joining a pendant edge to each rim vertex of $W_{n}$.

Definition 2.9

$G_{1} \odot G_{2}$ graph: the corona $G_{1}$ and $G_{2}$ of two graphs $G_{1}$ and $\mathrm{G}_{2}$ is defined as the graph procured by taking one copy of $\mathrm{G}_{1}$ and $n$ copies of $G_{2}$ and then joining the $i^{\text {th }}$ vertex of $G_{1}$ with an edge to every vertex in the $\mathrm{i}^{\text {th }}$ copy of $\mathrm{G}_{2}$.

- $P_{n} \odot K_{1}$ is called comb.

- $C_{\mathrm{n}} \odot K_{1}$ is a crown graph.

\section{MAIN RESULTS}

\section{Theorem 3.1.}

A ladder graph $P_{n} \mathrm{x} P_{2}$ is a QD graph.

Proof: Let $G=P_{n}$ x $P_{2}$ be a graph with $|V(G)|=2 n$ and $|E(G)|=3 n-2$.

The vertex and edge set is defined as

$V=\left\{u_{i}, v_{i}: i=0\right.$ to $\left.n\right\}$

$E=\left\{u_{i} v_{i}, u_{i} u_{i+1}, v_{i} v_{i+1} / 1 \leq i \leq n\right\}$.

and

Define the vertex labeling, we define an annexed function $f^{*}$ by classifying the graph $\mathrm{G}$ into two classes namely:

Case(1):

This case consists of the edges with both of their end vertices have labels either odd or even integers then the quad difference of the labels of the end vertices of each edge is an even number and these numbers form a strictly increasing sequence of even integers.

Case (2):

In this case, consider the set of edges, in which each edge has one vertex with odd integer as its label and the other end vertex with even integer as its label and the other end vertex with even integer as its label. Then the quad difference of the labels of the end vertices of each edge is an odd number and these numbers form a strictly increasing sequence of odd integers.

Also

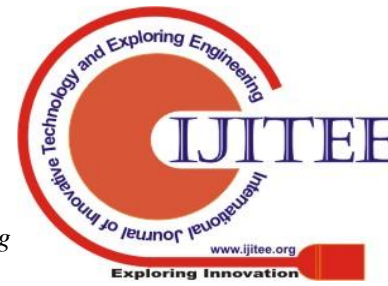


$f^{*}\left(e_{i}\right) \neq f^{*}\left(e_{j}\right)$ for any edge $e_{i}$ belongs to case (1) and any edge $e_{j}$ belongs to case (2); clearly it is seen that the induced function $f^{*}: E(G) \rightarrow N$ given by $f^{*}(u v)=\left|f(u)^{4}-f(v)^{4}\right|$ for all $u v \in E(G)$ is injective. Hence the ladder graph $L_{n}$ admits QDL.

Example 3.1 $P_{5} \times P_{2}$ is shown below in figure 3.1

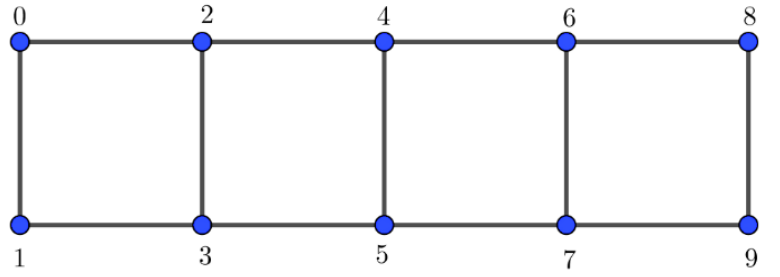

Figure 3.1

\section{Note 1.}

In the above theorem 3.1, joining the vertices of $u_{i} v_{i+1}$ by a new edge, we get triangular ladder namely $T L_{n}$ for $n \geq 2$, then the edge $u_{i} v_{i+1}$ receives the odd label with cardinality of vertices $2 n$ and $4 n-3$.

\section{Corollary 1.}

It is easily observed that $T L_{n}$ for $n \geq 2$, is a QDG.

Note 2.

In the theorem 3.1, joining the vertices of $u_{i} v_{i+1}$ and $v_{i} u_{i+1}$ by a new edges, we get diagonal triangular ladder namely $D L_{n}, n \geq 2$, then the newly formed edges receives the odd label with $|V(G)=2 n|$ and $E(G)=5 n-4$.

\section{Corollary 2.}

It is seen that $D L_{n}$ for $n \geq 2$, admits QDL.

\section{Theorem 3.2}

A fan graph $F_{n}, n \geq 2$ is a QDG.

\section{Proof:}

Let $G=F_{n}$ be a fan graph with $\left|V\left(F_{n}\right)\right|=n+1$ and $\left|E\left(F_{n}\right)\right|=2 n-1$.

The vertex set is defined as $\left\{u_{v} u_{i} / 0 \leq i \leq n\right\}$ and edge set $\left\{u u_{i}, u_{i} u_{i+1} / i=0\right.$ to $\left.n\right\}$.

The vertex labeling of a function is as follows:

$f(u)=0, f\left(u_{i}\right)=i$ for $1 \leq i \leq n$.

The edge labeling of the induced function $f^{*}$ is defined by $f^{*}\left(u u_{i}\right)=i^{4}, f^{*}\left(u_{i} u_{i+1}\right)=u_{i+1}^{4}-u_{i}^{4}$ for $1 \leq i \leq n$.

Also $\quad f^{*}\left(e_{i}\right) \neq f^{*}\left(e_{j}\right)$ for any edge $e_{i} e_{j}$, hence the induced function is injective. Hence the $\operatorname{graph} F_{n}$ admits QDL.

Example 3.2. QDL for $F_{n}$ is shown below in figure 3.2

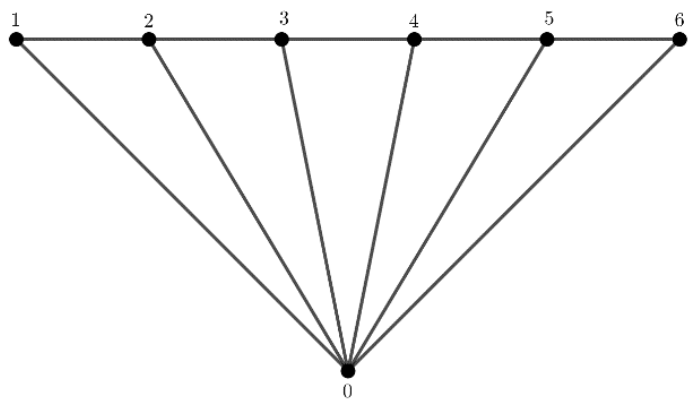

Figure 3.2
A friendship graph $T_{n}, n \geq 2$ is a QDG.

Proof:

Consider a graph $T_{n}$ with $\left|V\left(T_{n}\right)\right|=2 n+1$ and

$\left|E\left(T_{n}\right)\right|=3 n$.

Vertex and edge set is defined as

$V(G)=\left\{v_{0}, v_{i} / 1 \leq i \leq 2 n\right\}$ and

$E(G)=\left\{v_{0} v_{i}, v_{i} v_{i+1} / 1 \leq i \leq 2 n\right\}$

Now define a vertex labeling of a function mapping f from a vertex to $\{0,1,2, \ldots, 2 n\}$ as follows: $f\left(v_{0}\right)=0_{s} f\left(v_{i}\right)=i_{v} 1 \leq i \leq 2 n$.

A procured function is introduced for edge labeling as defined below for $1 \leq i \leq 2 n$

$f^{*}\left(v_{0} v_{i}\right)=i^{4}, f^{*}\left(v_{i} v_{i+1}\right)=v_{i+1}^{4}-v_{i}^{4}$.

Hence all the edge labeling defined are distinct and thus $f^{*}$ is injective.

Therefore $T_{n}$ satisfies QD graph.

Example 3.3. $T_{6}$ is illustrated below for QDL in figure 3.3

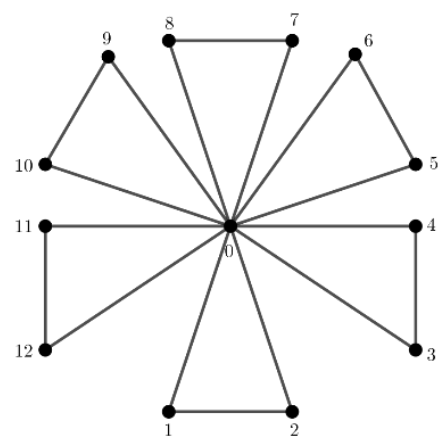

Figure 3.3

Theorem 3.4

A gear graph $G_{n}$ for $n \geq 3$ admits QDL.

Proof:

The graph $G_{n}$ has $2 n+1$ vertices and $3 n$ edges. Let $y_{0}$ be the apex vertex and $y_{1}, y_{2}, y_{1}, \ldots, y_{n}$ be the rim vertices of $W_{n}$ corresponding to $G_{n}$. Let $y_{1}^{s}, y_{2}, y_{2}^{s}, \ldots, y_{n}^{s}$ be the vertices of $G_{n}$ which makes subdivision of the edges of corresponding $W_{n}$, where $y_{i}^{s}$ is adjacent to $y_{i}$ and $y_{i+1}, i=1,2, \ldots, n-1$. $y_{n}$ is adjacent to $y_{n}$ and $y_{1}$.

We define a labeling function $f: V \rightarrow\{0,1,2, \ldots, 2 n\}$ as follows:

$f\left(y_{0}\right)=0, f\left(y_{i}\right)=2 i-1, f\left(y_{i}\right)=2 i$ for $1 \leq i \leq n$.

The edge labeling of induced function $f^{*}$ as follows:

$f^{*}\left(y_{0} y_{i}\right)=i^{4}$,

$f^{*}\left(Y_{i} y_{i}^{5}\right)$ and $f^{*}\left(Y_{i}^{5} Y_{i+1}\right)$ is labeled as same as the proof of theorem 3.1. Hence $f^{*}\left(e_{i}\right) \neq f^{*}\left(e_{j}\right)$ for any edge $e_{i} \neq e_{j}$. Therefore $f^{*}$ is injective and thus $G_{n}$ attains QD graph.

Example 3.4 : QDL for $G_{6}$ graph is shown below in figure 3.4

\section{Theorem 3.3}




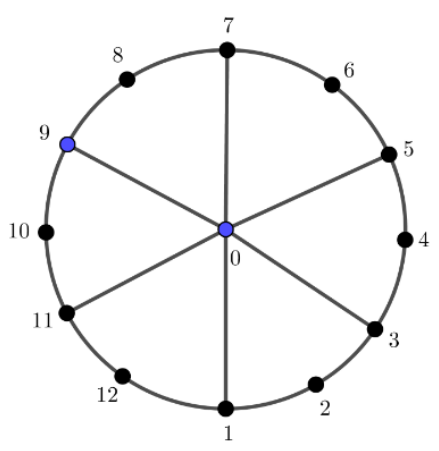

\section{Theorem 3.5}

Helm graph obtain QDG

\section{Proof:}

Consider a Helm graph with $2 n+1$ vertices and $3 n$ edges.

Let $\mathrm{x}_{0}$ be the apex vertex $x_{1}, x_{2}, \ldots \ldots \ldots x_{n}$ be the vertices and $x_{1}^{\prime}, x_{2}^{\prime}, x_{3}^{\prime}, \ldots ., x_{n}^{\prime}$ be the pendent vertices of Helm graph. We define the vertex labeling for as $f\left(x_{0}\right)=0 f\left(x_{0}\right)=2 i-1 f\left(x_{i}^{\prime}\right)=2 i, 1 \leq i \leq n$ and an induced function $f^{*}$ denoting is defined in proof of two cases in theorem in 2.1 and it is observed that all edge labeling are distinct and $f^{*}$ is injective.

Thus Helm graph provesQDL.

\section{Example 3.5}

A helm graph $H_{5}$ illustrated below in figure 3.5

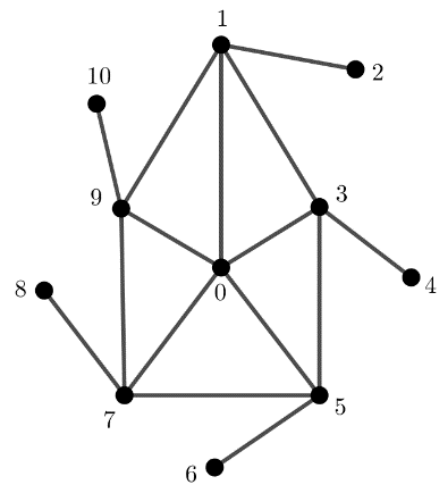

Figure 3.5

\section{Theorem 3.6}

A wheel graph $w_{n}=C_{n}+k_{1}$ for $n \geq 3$ admits QDL

\section{Proof :}

Let $G=w_{n}$ be a graph with $|v|=n+1 \&|E|=2 n$.

Let $p_{0}$ be apex vertex $\& p_{1}, p_{2}, \ldots \ldots, p_{n}$ be the recursive rim vertices of $w_{n}$.

Here we define the labeling function $f: v\left(w_{n}\right) \rightarrow\left\{0,1,2, \ldots \ldots v\left(w_{n} \mid\right\}\right.$ as follows:

$f\left(p_{0}\right)=0, f\left(p_{i}\right)=i, 1 \leq i \leq n_{x}$

So, from above defined function $f$, the induced fuction $f^{*}: E(G) \rightarrow N$ defined by

$f^{*}(u v)=\left|f(u)^{4}-f(v)^{4}\right|$ for every $u v \epsilon E(G)$ is injective. Hence wheel graph $w_{n}$ is Quad difference.

Example 3.6
Figure 3.4

$W_{12}=C_{12}+k_{1}$ is QDL shown in below figure 3.6

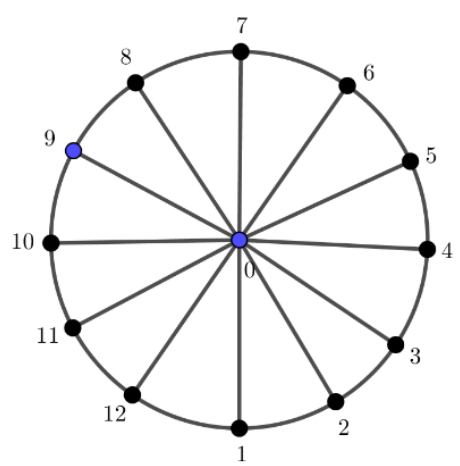

Figure 3.6

\section{Theorem3.7}

The graph $C_{n} \odot K_{1}$ admits QDL

Proof:

$|v|=|E|=2 n$. Define a vertex set $v=\left\{u_{i}, u_{i}^{s} / i=1\right.$ to

n $\}$ and edge set

$E=\left\{u_{i} u_{i}^{s}, u_{i} u_{i+1} / i=1\right.$ to $\left.\mathrm{n}\right\}$

Define a labeling $f: v \rightarrow\{0,1,2, \ldots \ldots n-1\}$ as follows $f\left(u_{i}\right)=2(i-1), f\left(u_{i}\right)=2 i-1$ for $1 \leq i \leq 2 n$.

The induced for $f^{*}$ for edge set labeling is defined as follows

$$
\begin{aligned}
& f^{*}\left(u_{i} u_{i}^{*}\right)=u_{i}^{4} u_{i}^{4} \\
& f^{*}\left(u_{i} u_{i+1}\right) \equiv 0 \bmod (8) \\
& f^{*}\left(u_{i} u_{i+1}\right)=(2 i)^{4}-[2(i-1)]^{4}
\end{aligned}
$$

Example 3.7: QDL of $C_{6} \odot K_{1}$ is given in the figure 3.7

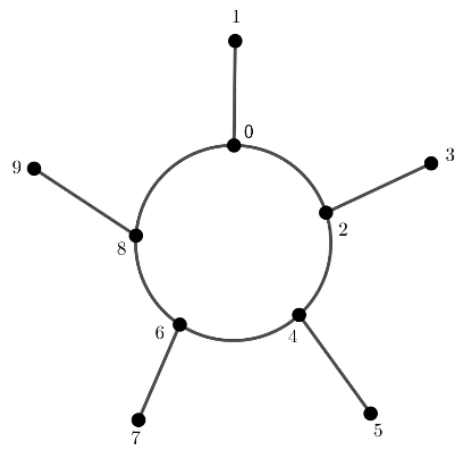

Figure 3.7

\section{Theorem 3.8}

The Dragon graph $D_{n}(m)$ admits QDL for $n \geq 3, m \geq 1$.

\section{Proof:}

Let $x_{1}, x_{2, m \ldots g} x_{n}$ be the vertices of the cycle $C_{n}$ and $x_{\mathrm{n}+1}, x_{2, \ldots \ldots s} x_{m}$ be the edges of the path $P_{m}$

The mapping $f: v\left(D_{n}(m)\right) \rightarrow\{0,1,2, \ldots \ldots n+m-1\}$ is defined by $f\left(x_{i}\right)=i_{x} 0 \leq i \leq n+m-1$ and the procured function $f^{*}: E(G) \rightarrow N$ is defined by $f^{*}\left(x_{i} x_{i+1}\right)=f\left(x_{i+1}\right)^{4}-f\left(x_{i}\right)^{4}$. Here the edge sets are $E_{1}=\left\{\left(x_{i} x_{i+1}\right) / 0 \leq i \leq n-1\right\}$

$E_{2}=\left\{x_{n-1} x_{0}\right\}$

$E_{a}=\left\{\left(x_{i} x_{n-1+i}\right) / n-1 \leq i \leq m\right\}$

And the edge labeling are

$f^{*}\left(x_{i} x_{i+1}\right)=\left|\left(x_{i+1}\right)^{4}-\left(x_{i}\right)^{4}\right|$ 
$f^{*}\left(x_{n-1} x_{0}\right)=(n-1)^{4}$

$f^{*}\left(x_{i} x_{n-1+i}\right)=\left(x_{i+1}\right)^{4}-\left(x_{i}\right)^{4}$ forn $-1 \leq i \leq m$.

Here the edges are distinct. Hence the dragon graph admits a QDL.

Example 3.9: The dragon graph $D_{4}(3)$ is a QDG in figure 3.9

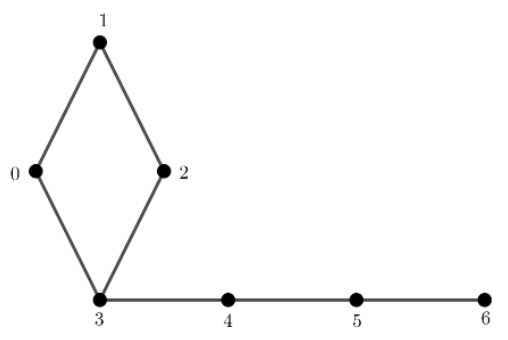

Figure 3.9

\section{CONCLUSION}

Here we have investigated the behavior of path, cycle and wheel related graphs like ladder, triangular ladder, diagonal ladder, fan, friendship, gear, helm, wheel and graphs satisfies QD labeling.

\section{REFERENCE}

[1]. Ajitha V, Arumugam S, Germina KA, “on square sum graphs", AKCE J. Graphs combin 2006, 6(1):1-10.

[2]. Gallian JA, A dynamics survey of graph labeling. The electronic journal of combinatorics 2009

[3]. Govindan V, Dhivya S, "Difference labeling of Jewel graph". International journal of mathematics trends and technology (IJMTT) - Volume 65, Issue 4-April/2019

[4]. Harary F, 1998, graph theory, Narosa publishing house, New Delhi, India

[5]. J. Shiama "cube difference labeling of some graphs", International journal of engineering science and innovative technology (IJESIT), Volume 2 , Issue 6-November/2013

[6]. J. Shiama, "Square difference labeling for Cartesian graph, International journal of computer applications (0975-08887), (44/4) (2012)

\section{AUTHORS PROFILE}

G. Subashini, Assistant Professor, Department of Science and Humanities, Bharath Institute of Higher Education and Research, India 